\title{
EFFECTIVE ION CHARGE
}

\author{
MAREK MONETA \\ University of Łódz, Chair of Solid State Physics \\ Pomorska 149, 90-236 Łódź, Poland
}

(Received March 15, 1995; revised version November 15, 1995; in final form February 13, 1996)

The impact parameter dependent energy transfer and random stopping power for ions carrying electrons were determined within the first-order Born approximation. The ion and atom were described by many-electron ground states. The excitations and ionizations of both collision partners were taken into account, but exchange of electrons was neglected. With the Bethe sum rule and closure relation, the random stopping was shown to have the Bethe form. For the Moliere form factors the analytical results were obtained. The effective charge was discussed in the random and channelling conditions. Comparison with some previous calculations was carried out.

PACS numbers: $34.50 . \mathrm{Bw}, 61.80 . \mathrm{Mk}$

\section{Introduction}

The basic processes responsible for the slowing down of fast heavy ions in matter are qualitatively understood [1-6], but the interest in this problem remains alive for at least two reasons. Ions with velocity $v$ proceed in the solid having, at least temporarily, bound electrons [7-11], even if $v>Z_{\text {atom }}^{2 / 3}$, and the distribution of charge is not completely predicted. Also, the contribution of higher order terms in the Born series $[12,13]$ creates a problem of completeness and validity of the starting point for simplified calculations. Experimental difficulties are caused by overlapping of the above and also other phenomena.

The problem of the random stopping power $S_{\mathrm{r}}$ for partially stripped ions has been formulated and solved within the first Born approximation [7-9]. Results of this calculation have shown rather good agreement with experiment [9], even without taking into account any excitation of the projectile. Calculations of the position-dependent stopping power for light ions specularly reflected from the surface [9] also have shown good agreement with experiment without projectile excitation.

In this paper we concentrate on presenting formulae for the impact parameter $b$ dependent average energy $\Delta E(b)$ transferred in a collision of an ion with an atom. 
The ion has been assumed to be relaxed to the ground state after each collision and can become, in a course of successive collisions with atoms, excited or ionised. In this meaning the loss of electrons has been included in the calculation, but the exchange has been neglected. The analytical results for $\Delta E(b)$ and $S_{\mathbf{r}}$ have been derived for the Moliere approximation to the Hartree-Fock form factor. A comparison of stopping and effective charge with the model neglecting excitation of the projectile was carried out.

Atomic units are used throughout this paper.

\section{Calculation procedure}

The time dependent Schrödinger equation written for the system of two colliding particles composed of nuclei and electrons is based on the following Hamiltonian:

$$
\begin{aligned}
& H=H_{0}+H_{\mathrm{I}}=H_{\mathrm{a}}+H_{\mathrm{i}}+H_{\mathrm{I}} \\
& H_{x}=\sum_{j=1}^{N_{x}}\left(-\frac{1}{2} \Delta_{j}-\frac{Z_{x}}{\left|\boldsymbol{R}_{j}^{x}\right|}+\frac{1}{2} \sum_{i=1}^{N_{x}} \frac{1}{\left|r_{j}-r_{i}\right|}\right), \\
& H_{\mathrm{I}}(b)=\frac{Z_{\mathrm{a}} Z_{\mathrm{i}}}{|\boldsymbol{R}|}-\sum_{a=1}^{N_{\mathrm{a}}} \frac{Z_{\mathrm{i}}}{\left|\boldsymbol{R}-r_{a}\right|}-\sum_{i=1}^{N_{\mathrm{i}}} \frac{Z_{\mathrm{a}}}{\left|\boldsymbol{R}-r_{i}\right|}+\sum_{a=1}^{N_{\mathrm{a}}} \sum_{i=1}^{N_{\mathrm{i}}} \frac{1}{\left|\boldsymbol{R}+r_{a}-r_{i}\right|}, \\
& H_{0}|m n\rangle=\omega_{m n}|m n\rangle, \quad R_{j}^{\mathrm{a}}=r_{\mathrm{a}}, \quad R_{j}^{\mathrm{i}}=\boldsymbol{R}+r_{j} .
\end{aligned}
$$

In the above subscript $x$ stands for atom (a) or ion (i) indices, and prime' excludes $i=j$ from the summation. $R$ is the separation of nuclei. $Z_{x}$ and $N_{x}$ are the atomic number and the number of electrons, respectively.

We assume the model in which the ion $\left(Z_{\mathrm{i}}, N_{\mathrm{i}}, n\right)$ follows the straight line trajectory $\boldsymbol{R}=\boldsymbol{b}+v t$ with the velocity $v$ and impact parameter $b$ in respect of the atom $\left(Z_{\mathrm{a}}, N_{\mathrm{a}}, m\right)$ located in the origin.

The Schrödinger equation can be written, in the standard manner, as the following set of differential equations:

$$
c_{m n}^{\prime}(t)=-\mathrm{i} \sum_{k l} \mathrm{e}^{\mathrm{i}} \omega_{m n k l} t\left\langle m n\left|H_{\mathrm{I}}\right| k l\right\rangle c_{k l}(t)
$$

where the summation $(k l)$ is taken over complete eigenstates of the unperturbed Hamiltonian $H_{0}$ belonging to the atom and the ion respectively, the prime' denotes time derivative, and $\omega_{m n 00}=\omega_{m n}-\omega_{00}$. The transition amplitude, $f_{m n}=c_{m n}(\infty)$ in the first-order perturbation treatment $\left(c_{00}(t) \approx 1, c_{k l}(t) \ll 1\right)$ is

$$
f_{m n 00}(b)=-\mathrm{i}\left\langle m n\left|\int_{-\infty}^{\infty} \mathrm{d} t \mathrm{e}^{\mathrm{i}} \omega_{m n 00} t H_{\mathrm{I}}(b)\right| 00\right\rangle .
$$

After substitution of $H_{\mathrm{I}}$ from Eq. (1) to Eq. (3) and use of the Fourier transform we get 


$$
\begin{aligned}
& f_{m n}(b)=\int \mathrm{d}^{3} q \frac{4 \pi}{q^{2}} \mathrm{e}^{-\mathrm{i} b q 2 \pi \delta\left(\omega_{m n 00}-q v\right)} \\
& \quad \times\left\langle m n\left|\left(Z_{\mathrm{a}}-\sum_{a=1}^{N_{\mathrm{a}}} \mathrm{e}^{\mathrm{i}} q r_{\mathrm{a}}\right)\left(Z_{\mathrm{i}}-\sum_{i=1}^{N_{\mathrm{i}}} \mathrm{e}^{-} \mathrm{i} q r_{i}\right)\right| 00\right\rangle .
\end{aligned}
$$

Under the model assumption that the eigenstates of the unperturbed Hamiltonian $H_{0}$ can be approximated by a product of the eigenstates of the components $\left\langle r_{\mathrm{a}} r_{\mathrm{i}} \mid m n\right\rangle=\left\langle r_{\mathrm{a}} \mid m\right\rangle\left\langle r_{\mathrm{i}} \mid n\right\rangle$, and consequently $\omega_{m n 00}=\omega_{m 0}+\omega_{n 0}$, we can write $f(b)$ in the form

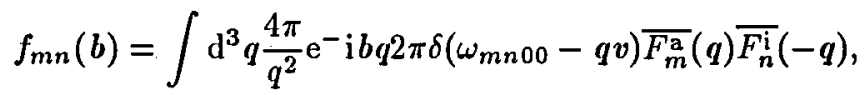

$$
\begin{aligned}
& \overline{F_{l}^{x}}(q)=\left\langle l_{x}\left|Z_{x}-\sum_{j=1}^{N_{x}} \mathrm{e}^{\mathrm{i}} q r_{j}\right| 0_{x}\right\rangle=Z_{x} \delta_{l 0}-F_{l}^{x}(q), \\
& F_{l}^{x}(q)=\left\langle l_{x}\left|\sum_{j=1}^{N_{x}} \mathrm{e}^{\mathrm{i}} q r_{j}\right| 0_{x}\right\rangle, \quad F^{x}(q)=F_{0}^{x}(q),
\end{aligned}
$$

and $x$ stands for atom (a) or ion (i).

With the use of the transition amplitude $f(b)$, Eq. (5), we can calculate the transition probability $P(b)$, the stopping power $S_{\mathrm{r}}$, and the impact parameter dependent energy loss $\Delta E(b)$, provided summation over appropriate final states can be performed, after integration over the Fourier space. For calculating $P(b)=$ $f^{+}(b) f(b)$ we can use the Fourier representations for each $f(b)$. After change of variables from $\left(q_{1}, q_{2}\right)$ to $(k, q)$, where $q_{1}-q_{2}=k, q_{1}+q_{2}=2 q$, and after some algebra we can make use of Eq. (5) to write $\left(Q_{m n}=\omega_{m n 00} / v\right)$ :

$$
\begin{aligned}
& P_{m n 00}(b)=(\pi v)^{-2} \int \mathrm{d}^{2} k \mathrm{e}^{-\mathrm{i} b k} \int_{Q m n}^{2 v} \frac{\mathrm{d}^{3} q \delta\left(q_{z}-\omega_{m n 00} / v\right)}{|q+k / 2|^{2}|q-k / 2|^{2}} \\
& \quad \times\left|Z_{m}^{\mathrm{a}}(k, q)\right|^{2}\left|Z_{n}^{\mathrm{i}}(k, q)\right|^{2}, \\
& \Delta E(b)=\sum_{m n} \omega_{m n 00} P_{m n 00}(b), \\
& S_{\mathrm{r}}=\int \mathrm{d}^{2} b \Delta E(b)=\frac{8 \pi}{v^{2}} \sum_{m n} \omega_{m n 00} \int_{Q m n}^{2 v} \frac{\mathrm{d} q}{q^{3}}\left|Z_{m}^{\mathrm{a}}(0, q)\right|^{2}\left|Z_{n}^{\mathrm{i}}(0, q)\right|^{2},
\end{aligned}
$$

where the functions

$$
\left|Z_{l}^{x}(k, q)\right|^{2}={\overline{F_{l}^{x}}}^{+}(q+k / 2) \overline{F_{l}^{x}}(q-k / 2)
$$

can be interpreted as the l-component of the ion $(x=\mathrm{i})$ or atom $(x=a)$ form factor.

These functions fulfil the following sum rules $[9,14-16]$ (see also Appendix A),

$$
\sum_{l} \omega_{l 0}^{x}\left|Z_{l}^{x}(k, q)\right|^{2}=\frac{1}{2}\left(q^{2}-\frac{1}{4} k^{2}\right) F^{x}(-k)
$$




$$
\begin{aligned}
& Z_{f x}^{2}(k, q)=\sum_{l}\left|Z_{l}^{x}(k, q)\right|^{2}=-Z_{x}\left[F^{x^{+}}\left(q+\frac{1}{2} k\right)+F^{x}\left(q-\frac{1}{2} k\right)\right] \\
& +\left\langle 0_{x}\left|\sum_{z \neq y}^{N_{x}} \sum_{y=1}^{N_{x}} \mathrm{e}^{\mathrm{i}}(q-k / 2) r_{z}-\mathrm{i}(q+k / 2) r_{y}\right| 0_{x}\right\rangle+Z_{x}^{2}+F^{x}(k), \\
& Z_{f x}^{2}(0, q)=\sum_{l}\left|Z_{l}^{x}(0, q)\right|^{2}=Z_{x}^{2}+N_{x}-2 Z_{x} F^{x}(q) \\
& +\left\langle 0_{x}\left|\sum_{z \neq y}^{N_{x}} \sum_{y=1}^{N_{x}} \mathrm{e}^{\mathrm{i}} q\left(r_{z}-r_{y}\right)\right| 0_{x}\right\rangle .
\end{aligned}
$$

We can interpret $Z_{f x}^{2}(k, q)$ as the total effective charge of the ion $(x=\mathrm{i})$, or the atom $(x=a)$ represented in the Fourier space. The summation over $l$ is taken over all the eigenstates (excited and ionised) of the ion or the atom separately. These sum rules can be treated as a generalization of the Thomas-Reiche-Kuhn sum rules. This can be seen, if we set $k=0$ in Eq. (11a) to obtain

$$
\sum_{l} \omega_{l 0}^{x}\left\langle 0_{x}\left|\sum_{j=1}^{N_{x}} \mathrm{e}^{-\mathrm{i} q r_{j}}\right| l_{x}\right\rangle\left\langle l_{x}\left|\sum_{i=1}^{N_{x}} \mathrm{e}^{\mathrm{i}} q r_{i}\right| 0_{x}\right\rangle=\frac{1}{2} q^{2} N_{x} .
$$

Following Bethe $[1,9,14,16]$, we use $S_{\mathbf{r}}$ of Eq. (9) to define a parameter $\omega$ such that the momentum $\omega / v$ separates the integration over $q$ in Eq. (9) into two regions, the dipole region, where the dipole expansion can be applied,

$$
S_{\mathrm{r}}^{\mathrm{D}}=\frac{8 \pi}{v^{2}} \sum_{m n} \omega_{m n 00} \int_{Q m n}^{\omega / v} \frac{\mathrm{d} q}{q^{3}}\left|Z_{m}^{\mathrm{a}}(0, q)\right|^{2}\left|Z_{n}^{\mathrm{i}}(0, q)\right|^{2}
$$

and the sum rule region, where the sum rules of Eq. (11) can be used, after exchange of integration over momentum space $q$ and summation over final states $(m n)$,

$$
S_{\mathrm{r}}^{\mathrm{S}}=\frac{8 \pi}{v^{2}} \sum_{m n} \omega_{m n 00} \int_{\omega / v}^{2 v} \frac{\mathrm{d} q}{q^{3}}\left|Z_{m}^{\mathrm{a}}(0, q)\right|^{2}\left|Z_{n}^{\mathrm{i}}(0, q)\right|^{2} .
$$

For the definition of $\omega$ we set $S_{\mathrm{r}}^{\mathrm{D}}(\omega)=0$, and then in consequence we obtain $S_{\mathrm{r}}=S_{\mathrm{r}}^{\mathrm{S}}$ for the total random stopping power. A similar treatment has been applied previously $[14,16]$. In the Bethe theory treating $S_{\mathrm{r}}$ for point charge projectiles $[1-4,16]$ and for the approach to $S_{\mathrm{r}}$ for nucleus-electrons composite staying in the ground state $[7,8]$, the momentum $\omega / v$ is cancelled out after adding the sum rule and the dipole contributions. The physical meaning of the parameter $\omega$ (originally Ref. [1] called it the mean excitation energy and denoted by $I$ ) is related to the logarithmic average of excitation energies of the excited system. Explicitly Eq. (12a) for $\omega$ takes the form

$$
\begin{aligned}
& \ln \omega\left[\left(Z_{\mathrm{a}}-N_{\mathrm{a}}\right)^{2} N_{\mathrm{i}}+\left(Z_{\mathrm{i}}-N_{\mathrm{i}}\right)^{2} N_{\mathrm{a}}\right] \\
& \quad=\left(Z_{\mathrm{a}}-N_{\mathrm{a}}\right)^{2} N_{\mathrm{i}} \ln \omega_{\mathrm{i}}+\left(Z_{\mathrm{i}}-N_{\mathrm{i}}\right)^{2} N_{\mathrm{a}} \ln \omega_{\mathrm{a}}+R / 2 v^{2} \\
& R=N_{\mathrm{i}}\left(\delta_{\mathrm{a}}+\sigma_{\mathrm{a}}\right)+N_{\mathrm{a}}\left(\delta_{\mathrm{i}}+\sigma_{\mathrm{i}}\right)=\omega^{2} N_{\mathrm{i}} N_{\mathrm{a}}, \quad \frac{1}{2} N_{x} \ln \omega_{x}=\sum_{k} \omega_{k 0} r_{k 0}^{2} \ln \omega_{k 0}
\end{aligned}
$$




$$
\begin{aligned}
& \sum_{n} r_{n 0}^{2}=N_{x}, \quad \sum_{n} \omega_{n 0} r_{n 0}^{2}=\frac{1}{2} N_{x}, \quad \sum_{n} \omega_{n 0}^{2} r_{n 0}^{2}=\delta_{x}, \\
& \sum_{n} \omega_{n 0}^{3} r_{n 0}^{2}=\sigma_{x}, \quad r_{n 0}^{2}=\left|\left\langle n\left|\sum_{j=1}^{N_{x}} e r_{j}\right| 0\right\rangle\right|^{2} .
\end{aligned}
$$

In the derivation of Eq. (13) we have used the sum rules obtained from Eqs. (24)-(27) of Ref. [15] after $k=0$ transition. The rules also were analysed in Ref. [16]. The parameter $\omega_{x}$ stands for Bethe's mean excitation energy $I_{x}$, for ion $(x=\mathrm{i})$ and atom $(x=a)$, respectively.

In the case of a neutral atom $\left(N_{\mathrm{a}}=Z_{\mathrm{a}}\right)$ and for high velocity bare ion $v \gg 1$ and $N_{\mathrm{i}}=0, \omega=\omega_{\mathrm{a}}$. If a neutral ion $\left(N_{\mathrm{i}}=Z_{\mathrm{i}}\right)$ collides with an atom $\left(N_{\mathrm{a}}=Z_{\mathrm{a}}\right)$, then according to Eq. (13), $\omega$ is determined by the equation $R=0$.

From the definition for the random stopping power $S_{\mathrm{r}}=\int \mathrm{d}^{2} b \Delta E(b)$ we assume that the parameter $\omega$ of Eq. (13) replaces the $\left\{\omega_{m n 00}\right\}$ set in the $\delta$-function of Eq. (7) for the impact parameter dependent electronic energy transfer $\Delta E(b)$. This step enables an exchange of the summation over final states $(m, n)$ of the IIamiltonian $H_{0}$ and the integration over momenta $k, q$ in Eq. (8). With the use of the sum rules, Eqs. (11), the summation over $(m, n)$ can be performed first to yield the $\Delta E(b)$ in a form consistent with integrated stopping power $S_{\mathrm{r}}$ of Eqs. $(9,12 b)$. The retained sum-rule contribution obtained after application of Eqs. (11a-c) in Eqs. (8), (9) can be written as

$$
\begin{aligned}
& S_{\mathrm{r}}=\frac{4 \pi}{v^{2}} \int_{\omega / v}^{2 v} \frac{\mathrm{d} q}{q}\left[N_{\mathrm{a}} Z_{f \mathrm{a}}^{2}(0, q)+N_{\mathrm{i}} Z_{f \mathrm{a}}^{2}(0, q)\right], \\
& \Delta E(b)=\frac{2}{(2 \pi v)^{2}} \int \mathrm{d}^{2} k \mathrm{e}^{-} \mathrm{i} k b \boldsymbol{b} \int_{\omega / v<|q|<2 v} \frac{\mathrm{d}^{3} q\left(q^{2}-\frac{1}{4} k^{2}\right) \delta(q z-\omega / v)}{\left|q+\frac{1}{2} k\right|^{2}\left|q-\frac{1}{2} k\right|^{2}} \\
& \quad \times\left[F^{\mathrm{a}}(k) Z_{f \mathrm{i}}^{2}(k, q)+F^{\mathrm{i}}(k) Z_{f \mathrm{a}}^{2}(k, q)\right] .
\end{aligned}
$$

If the ion is left in its ground state after the collision, then $n=0$ in Eqs. (7)-(9), and $\omega=\omega_{\mathrm{a}}$ follows from Eq. (13); Eqs. (14), (15) can thus be reduced to the form $[8,9]$, which retains only the screening effect

$$
\begin{aligned}
& S_{\mathrm{r}}=\frac{4 \pi}{v^{2}} \int_{\omega / v}^{2 v} \frac{\mathrm{d} q}{q} N_{\mathrm{a}}\left|Z_{\mathrm{i}}-F^{\mathrm{i}}(q)\right|^{2}, \\
& \Delta E(b)=\frac{2}{(2 \pi v)^{2}} \int \mathrm{d}^{2} k \mathrm{e}^{-} \mathrm{i} k b \int_{\omega / v<|q|<2 v} \frac{\mathrm{d}^{3} q\left(q^{2}-\frac{1}{4} k^{2}\right) \delta(q z-\omega / v)}{\left|q+\frac{1}{2} k\right|^{2}\left|q-\frac{1}{2} k\right|^{2}} \\
& \quad \times F^{\mathrm{a}}(k)\left[Z_{\mathrm{i}}-F^{\mathrm{i}}\left(q+\frac{1}{2} k\right)\right]\left[Z_{\mathrm{i}}-F^{\mathrm{i}}\left(q-\frac{1}{2} k\right)\right] .
\end{aligned}
$$

According to Eqs. (14), (15) the stopping is a sum of two components which are due to atom and projectile excitation and ionization, contrary to Eqs. (16), (17) where an ion-elastic collision with a frozen charge distribution on the projectile is assumed. This sum is a direct consequence of the model assumption that the eigenstate of the system is a product of component eigenstates, and the eigenenergy of the system is a sum of component eigenenergies. 


\section{Results and discussion}

The results based on Eqs. (16), (17) were discussed in Ref. [9] for the hydrogen-like one-electron ion form factor, $F^{\mathrm{i}}(q)=N_{\mathrm{i}} f(q)$, where $F(q)=$ $\left(1+q^{2} / q_{0}^{2}\right)^{-2}, q_{0}=2 / Z_{\mathrm{i}}, N_{\mathrm{i}}=1$. It was shown that the ion-elastic contribution was nearly sufficient for an appropriate description of stopping in the simplest case of one-electron helium and hydrogen ions scattered from a surface [9].

Apart from the contribution coming from ion (i) excitation and ionization the squares of the effective charge $Z_{f x}^{2}(k, q)$ in Eqs. (14), (15) have a slightly different behaviour. This can be seen when we replace in Eq. (11) the many-body form factors $F^{x}(q)$ by an incoherent sum of one-electron form factors $f^{x}(q)$ in the following manner:

$$
\begin{aligned}
& F^{x}(q) \Leftarrow N_{x} f^{x}(q), \quad\left\langle 0_{x}\left|\sum_{i \neq j}^{N_{x}} \mathrm{e}^{\mathrm{i}}(q-k / 2) r_{i}-\mathrm{i}(q+k / 2) r_{j}\right| 0_{x}\right\rangle \\
& \Leftarrow N_{x}\left(N_{x}-1\right) f^{x}\left(q+\frac{1}{2} k\right) f^{x}\left(q-\frac{1}{2} k\right) .
\end{aligned}
$$

The square of the effective charge, $Z_{f x}^{2}(k, q)$ of Eq. (11), is then given by

$$
\begin{aligned}
& Z_{f x}^{2}(k, q)=Z_{x}^{2}+N_{x} f^{x}(k)-Z_{x} N_{x}\left[f^{x}(q-k / 2)+f^{x}(q+k / 2)\right] \\
& \quad+N_{x}\left(N_{x}-1\right)\left[f^{x}(q-k / 2) f^{x}(q+k / 2)\right] \\
& Z_{f x}^{2}(0, q)=\left\{Z_{x}+N_{x} f^{x}(q)\right\}^{2}+N_{x}\left\{1-\left[f^{x}(q)\right]^{2}\right\}
\end{aligned}
$$

where $f^{x}(q)$ is the one-electron form factor of the ion $(x=\mathrm{i})$ or atom $(x=\mathrm{a})$.

On analysing $Z_{f \mathrm{i}}^{2}(0, q)$ for the ion we can distinguish the screening $\left[Z_{\mathrm{i}}-N_{\mathrm{i}} f^{\mathrm{i}}(\boldsymbol{q})\right]^{2}$ and the anti-screening $N_{\mathrm{i}}\left\{1-\left[f^{\mathrm{i}}(q)\right]^{2}\right\}$ contributions $[5,17]$. The screening of the ion is observed at large impact parameter $b$ (corresponding to small momentum transfer $q$ ) as $Z_{f \mathrm{i}}^{2}(0,0)=\left\{Z_{\mathrm{i}}-N_{\mathrm{i}}\right\}^{2}$ in accordance with the frozen-charge state model of Eq. (16). The anti-screening is caused by ionic electrons at small $b$ (large $q$ ) and results in $Z_{f \mathrm{i}}^{2}(0, \infty)=Z_{\mathrm{i}}^{2}+N_{\mathrm{i}}$ in disagreement with $Z_{\mathrm{i}}^{2}$ of Eq. (16) and the point charge model. Then both mechanisms strengthen close collisions over distant collisions. Apart from this contribution coming from excitation and ionization of an atom by an ion, there is also a second one component in Eqs. (14), (15) coming from excitation of the ion (represented by $N_{\mathrm{i}}$ and $\left.F^{\mathrm{i}}(k)\right)$ by the atomic charge $Z_{f \mathrm{a}}^{2}(k, q)$. This component must be also analysed in terms of screening and anti-screening phenomena.

In order to obtain analytical results for $\Delta E(b)$ and $S_{\mathrm{r}}$ of Eqs. (14), (15), we simplify conditions as much as possible. We make an advantage of Eq. (19) with the Moliere approximation [8] to the Hartree-Fock form factor describing both ion and atom

$$
f^{x}(q)=\sum_{j=1}^{3} \alpha_{j}\left(1+q^{2} / \gamma_{j}^{2}\right), \quad \gamma_{j}=\beta_{j} / a_{\mathrm{TF}}
$$




$$
\alpha_{j}=(0.1,0.55,0.35), \quad \beta_{j}=(6.0,1.2,0.3), \quad a_{\mathrm{TF}}=0.8853 Z_{x}^{-1 / 3} .
$$

This approximation assumes a statistical description of the many-electron system corresponding to a screening parameter $a_{\text {TF }}$.

$S_{\mathbf{r}}$ of Eq. (14) and $\Delta E(b)$ of Eq. (15) can be rewritten, respectively, as

$$
\begin{aligned}
& S_{\mathrm{r}}=\frac{4 \pi}{v^{2}}\left[N_{\mathrm{a}} S_{\mathrm{ai}}(0)+N_{\mathrm{i}} S_{\mathrm{ia}}(0)\right], \\
& \Delta E(b)=\frac{\pi}{v^{2}} \int \mathrm{d}^{2} k e^{-\mathrm{i} k b}\left[N_{\mathrm{a}} f^{\mathrm{a}}(k) S_{\mathrm{ai}}(k)+N_{\mathrm{i}} f^{\mathrm{i}}(k) S_{\mathrm{ia}}(k)\right], \\
& S_{y x}(k)=\frac{1}{2 \pi} \int_{\omega / v<|q|<2 v} \frac{\mathrm{d}^{3} q\left(q^{2}-\frac{1}{4} k^{2}\right) \delta\left(q_{z}-\omega / v\right)}{\left|q+\frac{1}{2} k\right|^{2}\left|q-\frac{1}{2} k\right|^{2}} Z_{f x}^{2}(k, q) .
\end{aligned}
$$

In $S_{y x}(k)$ the dependence on $y$ is incorporated in $\omega$ of Eq. (13). In Eqs. (21), (22) the ion stopping is clearly separated into two components. In the first one the ion, represented by the function $S_{\mathrm{ai}}(k)$ of ion effective charge $Z_{f \mathrm{i}}^{2}(k, q)$, excites the atom, represented by atomic form factor $N_{\mathrm{a}} f^{\mathrm{a}}(k)$. In the second component the situation is opposite, ionic electrons are excited by the effective charge of the atom. This splitting is a direct consequence of the assumption that the ground state of the ion-atom composite is a product of the component eigenstates and the eigenenergy is a sum of corresponding eigenenergies.

For $Z_{f x}^{2}(k, q)$ given by Eq. (19) and $f^{x}(q)$ by Eq. $(20)$, i.e. by the Moliere approximation to the Hartree-Fock form factor, the integral for $S_{y x}(k)$ of Eq. (23) can be calculated analytically with the result given in Appendix B, Eqs. (B1), (B2). This analytical form of $S_{y x}(k)$ is the basic result of this work.

We can make the following simplifications which may provide some instructive conclusions. Let us assume that the ion of atomic number $Z_{\mathrm{i}}$ carrying $N_{\mathrm{i}}$ electrons is retained in its ground state $(n=0)$ after collision with a neutral atom. We use $S_{y x}(k)$ of Eq. (23), in the small $k$ approximation $\left(S_{y x}(0)\right.$ of Eq. (B2)). The physical meaning of this approximation is related to the fact that in the calculation of the random stopping $S_{\mathrm{r}}=\int \mathrm{d}^{2} b \Delta E(b)$, in consequence of the $\delta(k)$-function, the whole contribution comes from the $k=0$ region. Then in calculating $\Delta E(b)$ we assume that only this region contributes significantly to $S_{y x}(k)$. It should be noted that the condition $k=0$ is not directly related to the dipole approximation and large impact parameter collisions (see discussion in Ref. [3]). After the integration over the whole momentum $k$ space the analytical result for the stopping power $S_{\mathbf{r}}$ given by Eq. (21) and for the energy transfer $\Delta E(b)$ given by Eq. (22) is

$$
\begin{aligned}
& S_{\mathrm{r}}=\frac{4 \pi}{v^{2}} N_{\mathrm{a}} S_{\mathrm{ai}}(0), \\
& \Delta E(b)=\frac{1}{2 \pi} S_{\mathrm{r}} \sum_{m=1}^{3} \alpha_{m} \gamma_{m}^{2} K_{0}\left(\gamma_{m} b\right),
\end{aligned}
$$

where $K_{0}(x)$ is the Bessel function of the second kind and $\alpha_{m}$ and $\gamma_{m}$ are the atomic parameters of Eq. (20) and

$$
S_{\mathrm{ai}}(0)=\left(Z_{\mathrm{i}}-N_{\mathrm{i}}\right)^{2} \ln \left|\frac{2 v^{2}}{\omega}\right|
$$




$$
\begin{aligned}
& +2 Z_{\mathrm{i}} N_{\mathrm{i}} \sum_{m=1}^{3} \alpha_{m} \varphi_{\mathrm{i}}\left(\gamma_{m}^{2}\right)-N_{\mathrm{i}}\left(N_{\mathrm{i}}-1\right)\left\{\sum_{j=1}^{3} \alpha_{j}^{2}\left[\phi_{\mathrm{i}}\left(\gamma_{j}^{2}\right)-\psi_{\mathrm{i}}\left(\gamma_{j}^{2}, \gamma_{j}^{2}\right)\right]\right. \\
& \left.-\sum_{j \neq k}^{3} \sum_{k=1}^{3} \alpha_{j} \alpha_{k}\left(\gamma_{j}^{2}-\gamma_{k}^{2}\right)^{-1}\left[\phi_{\mathrm{i}}\left(\gamma_{j}^{2}\right) \gamma_{k}^{2}-\phi_{\mathrm{i}}\left(\gamma_{k}^{2}\right) \gamma_{j}^{2}\right]\right\}
\end{aligned}
$$

where the functions $\phi_{\mathrm{i}}(x)$ and $\psi_{\mathrm{i}}(x)$ determined by the ionic parameters $\alpha$ and $\gamma$ of Eq. (20) are given in Appendix B. The effective charge $Z_{\text {eff }}^{2}(b)$, defined as the energy lost by an ion carrying electrons (and converted into the excitation and ionization of the atom), related to the energy lost by the proton is

$$
Z_{\text {eff }}^{2}(b)=\Delta E_{\text {ion }}(b) / \Delta E_{\text {proton }}(b) .
$$

For an elastic collision of an $N_{\mathrm{i}}$-electron ion with an atom $Z_{\mathrm{eff}}^{2}(b)$ is

$$
Z_{\mathrm{eff}}^{2}(b)=Z_{\mathrm{eff}}^{2}=S_{\mathrm{ai}}^{\mathrm{ion}}(0) / S_{\mathrm{ai}}^{\text {proton }}(0),
$$

where, from Appendix $\mathrm{B}, S_{\mathrm{ai}}^{\text {proton }}(0)=\phi_{\mathrm{i}}(0)=\ln \left|2 v^{2} / \omega_{\mathrm{a}}\right|$ for the proton. It is clearly seen that $Z_{\text {eff }}^{2}(b)$ of Eq. (27) is independent of $b$. This is a direct consequence of the accepted approximation (i.e. $k=0$ ) and of the assumption that the ion is not excited in the collision. In this case the ion acts on the atom (exciting and ionising it) only as a certain stable distribution of charge and therefore proportional to the action exerted on the atom by proton at the same impact parameter.

Generally, if we do not use simplifying approximations, even in the simplest, spherically symmetric ground state of the atom, the one-dimensional integration in Eq. (22) has to be performed numerically.

The function of $Z_{\mathrm{eff}}^{2} / Z_{\mathrm{i}}^{2}$ for ions of atomic number $Z_{\mathrm{i}}$, carrying $N_{\mathrm{i}}$ electrons in an ion-elastic collision with $\mathrm{Al}$ atoms $\left(Z_{\mathrm{a}}=13, \omega_{\mathrm{a}}=6\right)$ was determined. The results of Eq. (26) for the Moliere form factor and for ion velocities $v$ ranging from 2 to 10 were drawn in Fig. 1. We choose the Moliere approximation because of its simplicity which enables analytical calculations and due to the fact that it gives roughly the correct behaviour for the form factor of heavier atoms. However, the validity of this approximation is restricted due to the screening parameter, $a_{\mathrm{TF}}$, of Eq. (20), which reflects the statistical approach to the description of the atom.

The increase of $Z_{\mathrm{eff}}^{2} / Z_{\mathrm{i}}^{2}$ with $Z_{\mathrm{i}}$ means, for efficiency of excitations of atomic electrons, that the screening of the ionic nucleus by the electron plays a major role at low $Z_{\mathrm{i}}$ and a minor one at higher values. This is quite opposite to the sharp decrease in experimental $Z_{\text {eff }}^{2} / Z_{\mathrm{i}}^{2}$ values with $Z_{\mathrm{i}}$ summarized in semi-empirical formula [19]. In this meaning, the one-electron model for a high $Z_{\mathrm{i}}$ ion beam is here of restricted applicability, since statistically in a beam the high $Z_{\mathrm{i}}$ ion tends to bind more than one electron, causing a rapid decrease in the excitation efficiency. For comparison the results for hydrogen-like one-electron form factors [9] are drawn in Fig. 1 separately, showing the same overall dependence upon $Z_{\mathrm{i}}$ but a lower absolute value. The dependence of $Z_{\text {eff }}^{2} / Z_{\mathrm{i}}^{2}$ upon the parameter $\omega$, of Eq. (13), is shown in Fig. 2 for an ion of atomic number $Z_{\mathrm{i}}=10$ moving with velocities ranging from $v=2$ to $v=10$. The function changes rapidly with $\omega$ for low $v$ and $\omega$, while for higher $v$ and $\omega$ the dependence is weaker. The crossing point 

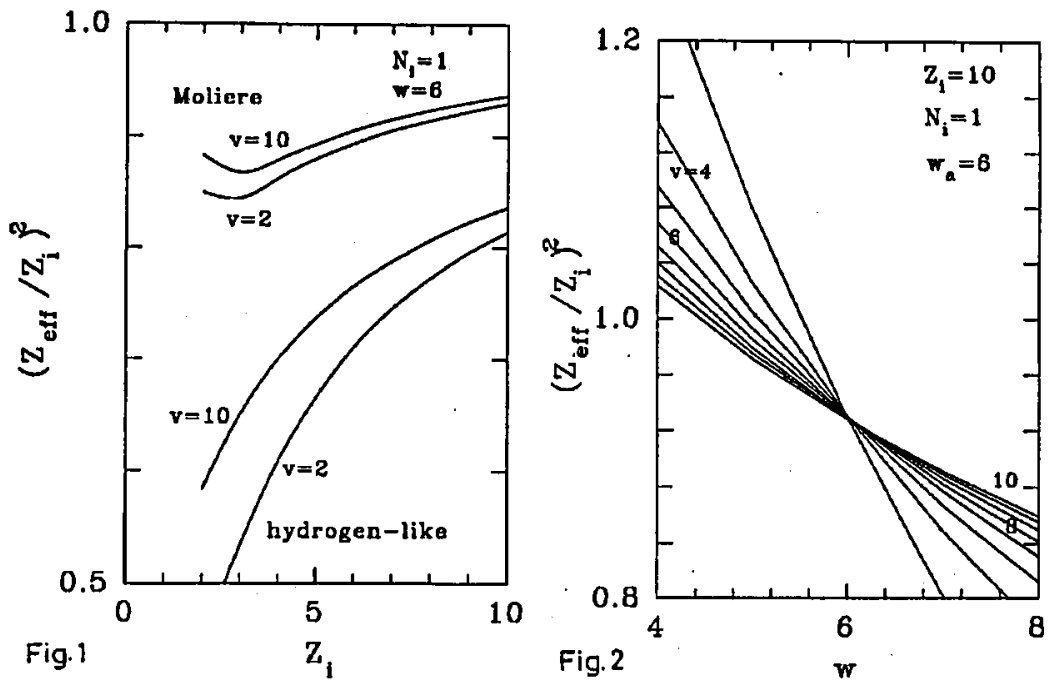

Fig. 1. The effective charge squared $Z_{\text {eff }}^{2} / Z_{\mathrm{i}}^{2}$ of Eq. (26), for ions of atomic number $Z_{\mathrm{i}}$ carrying one electron $\left(N_{\mathrm{i}}=1\right)$ in an ion-elastic collision with an $\mathrm{Al}$ atom $\left(Z_{\mathrm{a}}=13\right.$, $\left.\omega_{a}=6\right)$. The upper two curves are for the Moliere form factor, Eq. (20), and the two lower are calculated for the hydrogen-like form factor within the shellwise approach of Ref. [9].

Fig. 2 The $Z_{\text {eff }}^{2} / Z_{i}^{2}$ of Eq. (26), for ions of atomic number $Z_{\mathrm{i}}=10$ carrying one electron $\left(N_{\mathrm{i}}=1\right)$ in an ion-elastic collision with an $\mathrm{Al}$ atom $\left(Z_{\mathrm{a}}=13, \omega_{\mathrm{a}}=6\right)$ in dependence upon the parameter $\omega$. The form factors of Eq. (20) were used.

is close to $\omega=6$, the mean excitation energy for an $\mathrm{Al}$ atom. For the parameter $\omega=\omega_{\mathrm{a}}$ the dependence of $Z_{\mathrm{eff}}^{2} / Z_{\mathrm{i}}^{2}$ on the number of electrons $N_{\mathrm{i}}$ bound to the ion is shown in Fig. 3, again for $Z_{\mathrm{i}}=10$. The minimum of the stopping was found for $N_{\mathrm{i}}=3-4$, in the velocity range $v=3-10$. This effect can be related to the similar minimum of the random stopping power $S_{\mathrm{r}}$ as shown by Kim and Cheng [7].

According to Eq. (26), derived for the Moliere atomic and ionic form factors and with the use of the small $k$ form for $S_{y x}(k)$, the function of the $Z_{\mathrm{eff}}^{2} / Z_{i}^{2}$ is independent of the impact parameter $b$. Also, as shown in Fig. 4, the effective charge only weakly depends on the ion velocity $v$ when $\omega=\omega_{\mathrm{a}}$, rises sharply for $\omega>\omega_{\mathrm{a}}$ and decreases otherwise. This may be related to the results of the shellwise treatment presented in Ref. [9], which was based on the hydrogen-like form factors. Also from experiment [19], a sharp increase in $Z_{\text {eff }}^{2} / Z_{\mathrm{i}}^{2}$ with $v$ can be obtained, suggesting that for the system composed of an $\mathrm{Al}$ atom and a projectile of $Z_{\mathrm{i}}=10$ with one electron $N_{\mathrm{i}}=1$ the parameter $\omega$ of Eq. (13) should be rather greater than $\omega_{\mathrm{a}}$.

Theoretically the dependence on $b$ and $v$ is caused mainly by differences in the efficiency of excitation and exchange of electrons from inner and outer shells [18]. The experimental result is influenced by processes of dynamic electron exchange in the ion beam, by surface effects and many others which cause that results of 


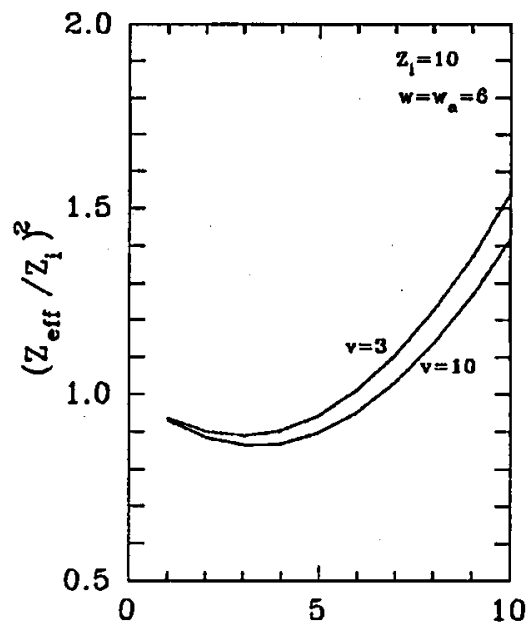

Fig. 3

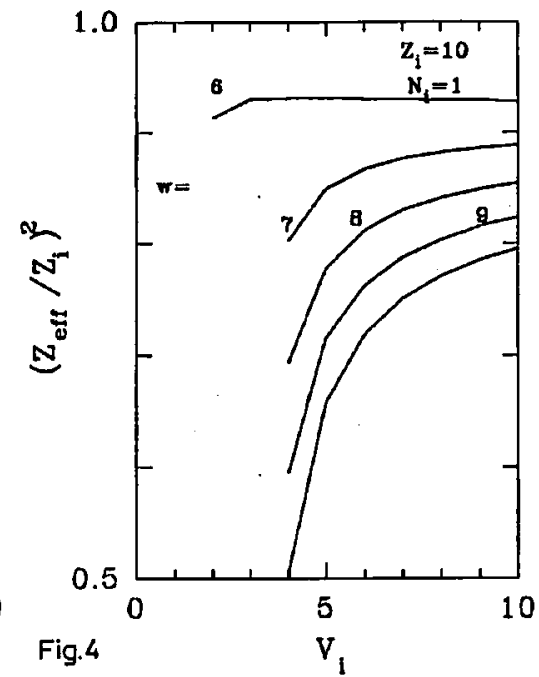

Fig. 3. The dependence of $Z_{\text {eff }}^{2} / Z_{i}^{2}$, Eq. (26), upon the number of carried electrons $N_{\mathrm{i}}$ for ions of atomic number $Z_{\mathrm{i}}=10 \mathrm{in}$ an ion-elastic collision with $\mathrm{Al}$ atom $\left(Z_{\mathrm{a}}=13\right.$, $\omega_{\mathrm{a}}=6$ ) for ion velocities $v=3$ and $v=10$.

Fig. 4 The velocity $v$ dependence of $Z_{\text {eff }}^{2} / Z_{i}^{2}$, Eq. (26), for ions of atomic number $Z_{\mathrm{i}}=10$ carrying electron in an ion-elastic collision with $\mathrm{Al}$ atom $\left(Z_{\mathrm{a}}=13, \omega_{\mathrm{a}}=6\right)$.

calculations based on such a simplified model of one-electron ion are not directly comparable with experiment.

In conclusion, the definition of the mean excitation energy $\omega, S_{\mathrm{r}}(\omega)=0$ for a bare ion in the random case, seems to be a useful tool for a unified treatment of the random and impact parameter problems, and also for ions carrying electrons. The validity of this extension, which has to be discussed separately, is confined by the request that the relation $S_{\mathrm{r}}=\int \mathrm{d}^{2} b \Delta E(b)$ must be fulfilled.

Within this approach, the analytical result for $S_{y x}(k)$ can be obtained provided we replace, as in Eq. (18), the many-body form factors describing both ion and atom by the incoherent sum of one-electron form factors. The Moliere approximation to the Hartree-Fock form factor was used here. An analytical result, although much more complicated, can be also obtained for hydrogen-like one-electron form factor, the result applicable for ions with a small number of electrons. If $S_{y x}(k)$ and form factors are spherically symmetric, then the final one-dimensional integral over $k$ in $\mathrm{Eq}$. (22) has to be performed numerically.

\section{Appendix A}

In order to show the validity of Eq. (11a), i.e.

$$
\sum_{l} \omega_{l 0}^{x}\left|Z_{l}^{x}(k, q)\right|^{2}=\frac{1}{2}\left(q^{2}-\frac{1}{4} k^{2}\right) F^{x}(-k),
$$

we follow the derivation by Esbensen and Golovchenko [3] given for a system of 
$N_{x}$ electrons which is described by the Hamiltonian

$$
H_{x}=\sum_{i=1}^{N_{x}} \frac{1}{2} \Delta_{i}+V\left(r_{1}, \ldots r_{N_{x}}\right), \quad H_{x}\left|l_{x}\right\rangle=\omega_{l}^{x}\left|l_{x}\right|, \quad \sum_{l_{x}}\left|l_{x}\right\rangle\left\langle l_{x}\right|=1 .
$$

Let us substitute the expression for $Z_{f x}^{2}(k, q)$ of Eq. (10) with the definition of $\overline{F_{l}^{x}}(q)$ from Eq. (5) to get

$$
\begin{aligned}
& x_{1}=q-\frac{1}{2} k, \quad x_{2}=q+\frac{1}{2} k, \\
& \sum_{l}=\sum_{l} \omega_{l 0}^{x} \mid Z_{l}^{x}\left(k,\left.q\right|^{2}=\sum_{l} \omega_{l 0}^{x} \overline{F_{l}^{x}}+\left(x_{2}\right) \overline{F_{l}^{x}}\left(x_{1}\right)\right. \\
& =\sum_{l} \omega_{l 0}^{x}\left\langle 0_{x}\left|\sum_{i=1}^{N_{x}} \mathrm{e}^{-\mathrm{i} x_{2} r_{i}}\right| l_{x}\right\rangle\left\langle l_{x}\left|\sum_{j=1}^{N_{x}} \mathrm{e}^{\mathrm{i}} x_{1} r_{j}\right| 0_{x}\right\rangle \\
& =\sum_{l}\left\langle 0_{x}\left|\sum_{i=1}^{N_{x}} \mathrm{e}^{-\mathrm{i} x_{2} r_{i}}\right| l_{x}\right\rangle\left\langle l_{x}\left|\left[H_{x}, \sum_{j=1}^{N_{x}} \mathrm{e}^{\mathrm{i} x_{1} r_{j}}\right]\right| 0_{x}\right\rangle \\
& =\left\langle 0_{x}\left|\sum_{i=1}^{N_{x}} \mathrm{e}^{-\mathrm{i} x_{2} r_{i}}\left[H_{x}, \sum_{j=1}^{N_{x}} \mathrm{e}^{\mathrm{i} x_{1} r_{j}}\right]\right| 0_{x}\right\rangle \\
& =\frac{1}{2}\left\langle 0_{x}\left|\sum_{i=1}^{N_{x}} \sum_{j=1}^{N_{x}} \mathrm{e}^{\mathrm{i}} x_{1} r_{j}-\mathrm{i} x_{2} r_{i}\left(x_{1}^{2}-2 \mathrm{i} x_{1} \cdot \nabla_{j}\right)\right| 0_{x}\right\rangle \text {. }
\end{aligned}
$$

If we assume additionally that the ground state function is chosen real, i.e. $\left\langle\boldsymbol{r}_{1}, \boldsymbol{r}_{N_{x}} \mid 0_{x}\right\rangle=\phi_{0}\left(\boldsymbol{r}_{1}, \boldsymbol{r}_{N_{x}}\right)$ we can write

$$
\begin{aligned}
0= & \int \mathrm{d}^{3} r_{j} \nabla_{j}\left[\mathrm{e}^{\mathrm{i}}\left(x_{1} r_{j}-x_{2} r_{i}\right) \mathrm{i} x_{1} \phi_{0}^{2}\left(r_{1}, r_{N_{x}}\right)\right] \\
& =\int \mathrm{d}^{3} r_{j} \mathrm{e}^{\mathrm{i}}\left(x_{1} r_{j}-x_{2} r_{i}\right) \mathrm{i} x_{1} \cdot \nabla_{j} \phi_{0}^{2}\left(r_{1}, r_{N_{x}}\right) \\
& +\int \mathrm{d}^{3} r_{j} \mathrm{e}^{\mathrm{i}}\left(x_{1} r_{j}-x_{2} r_{i}\right) \mathrm{i} x_{1}\left(\mathrm{i} x_{1}-\mathrm{i} x_{2} \delta_{i j}\right) \phi_{0}^{2}\left(r_{1}, r_{N_{x}}\right) .
\end{aligned}
$$

Substituting the component with gradient (A2) into (A1) we find

$$
\begin{aligned}
& \sum_{l}=\frac{1}{2} \sum_{i j} \int \mathrm{d}^{3} r_{1} \int \mathrm{d}^{3} r_{N_{x}} \mathrm{e}^{\mathrm{i}}\left(x_{1} r_{j}-x_{2} r_{i}\right) \\
& \times\left[\mathrm{i} x_{1}^{2}+\mathrm{i} x_{1}\left(\mathrm{i} x_{1}-\mathrm{i} x_{2} \delta_{i j}\right)\right] \phi_{0}^{2}\left(r_{1}, r_{N_{x}}\right) \\
& =\frac{1}{2} x_{1} x_{2} \sum_{i} \int \mathrm{d}^{3} r_{1} \int \mathrm{d}^{3} r_{N_{x}} \mathrm{e}^{\mathrm{i}}\left(x_{1}-x_{2}\right) r_{i} \phi_{0}^{2}\left(r_{1}, r_{N_{x}}\right) \\
& =\frac{1}{2} x_{1} x_{2}\left\langle 0_{x}\left|\sum_{i} \mathrm{e}^{\mathrm{i}}\left(x_{1}-x_{2}\right) r_{i}\right| 0_{x}\right\rangle \text {. }
\end{aligned}
$$


And finally we obtain

$$
\begin{aligned}
\sum_{l} & =\sum_{l} \omega_{l 0}^{x}\left|Z_{l}^{x}(k, q)\right|^{2}=\frac{1}{2}\left(q^{2}-\frac{1}{4} k^{2}\right)\left\langle 0_{x}\left|\sum_{j=1}^{N_{x}} \mathrm{e}^{-\mathrm{i} k r_{j}}\right| 0_{x}\right\rangle \\
& =\frac{1}{2}\left(q^{2}-\frac{1}{4} k^{2}\right) F^{x}(-k)
\end{aligned}
$$

in accordance with Eq. (11a).

A derivation for $Z_{f x}^{2}(k, q)=\sum_{l}\left|Z_{l}^{x}(k, q)\right|^{2}$ of Eq. (11b) can be obtained by a straightforward substitution of $\left|Z_{l}^{x}(k, q)\right|^{2}=\overline{F_{l}^{x}}(q+k / 2) \overline{F_{l}^{x}}(q-k / 2)$ of Eq. (10) and by using the closure relation to yield

$$
\begin{aligned}
& Z_{f x}^{2}(k, q)=\sum_{l} \overline{F_{l}^{x}}\left(q+\frac{1}{2} k\right) \overline{F_{l}^{x}}\left(q-\frac{1}{2} k\right) \\
& =\sum_{l}\left\langle 0_{x}\left|Z_{x}-\sum_{i=1}^{N_{x}} \mathrm{e}^{\mathrm{i}}\left(q+\frac{1}{2} k\right) r_{i}\right| l_{x}\right\rangle\left\langle l_{x}\left|Z_{x}-\sum_{j=1}^{N_{x}} \mathrm{e}^{\mathrm{i}}\left(q-\frac{1}{2} k\right) r_{j}\right| 0_{x}\right\rangle
\end{aligned}
$$

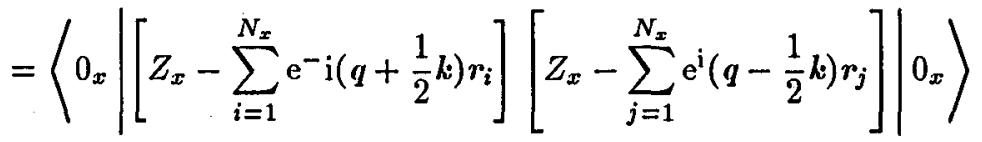

$$
\begin{aligned}
& =Z_{x}^{2}+F^{x}(k)-Z_{x}\left[F^{x^{+}}\left(q+\frac{1}{2} k\right)+F^{x}\left(q-\frac{1}{2} k\right)\right]
\end{aligned}
$$

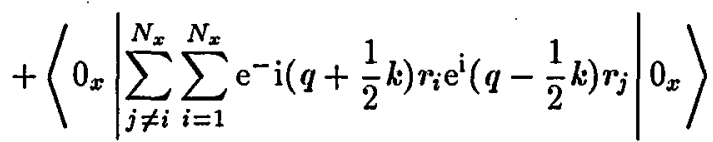

in accordance with Eq. (11b).

\section{Appendix B}

For $Z_{f x}^{2}(k, q)$ given by Eq. (19) and $f^{x}(k)$ by Eq. (20), the integral $S_{y x}(k)$ prescribed by Eq. (23) can be calculated analytically with the result

$$
\begin{aligned}
& S_{y x}(k)=\left[Z_{x}^{2}+N_{x} f^{x}(k)\right]\left[\phi_{x}(0)-\psi_{x}(0,0)\right]-2 Z_{x} N_{x}\left\{\frac{1}{2} \phi_{x}(0)\right. \\
& \left.-\psi_{x}(0,0)+\sum_{i=1}^{3} \frac{1}{2} \alpha_{i}\left[\psi_{x}\left(0, \frac{\gamma_{i}^{2}}{2}\right)+\psi_{x}\left(\gamma_{i}^{2}, \frac{\gamma_{i}^{2}}{2}\right)-\phi_{x}\left(\gamma_{i}^{2}\right)\right]\right\} \\
& +N_{x}\left(N_{x}-1\right)\left\{-\psi_{x}(0,0)+\sum_{i}^{3} \sum_{i}^{3} \frac{1}{2} \alpha_{i} \alpha_{j}\right. \\
& \times\left[\psi_{x}\left(\gamma_{i}^{2}, \frac{\gamma_{i}^{2}}{2}\right)+\psi_{x}\left(\gamma_{j}^{2}, \frac{\gamma_{j}^{2}}{2}\right)+\psi_{x}\left(0, \frac{\gamma_{i}^{2}}{2}\right)+\psi_{x}\left(0, \frac{\gamma_{j}^{2}}{2}\right)\right. \\
& \left.\left.-\psi_{x}\left(\gamma_{i}^{2}, \frac{\gamma_{i}^{2}+\gamma_{j}^{2}}{2}\right)-\psi_{x}\left(\gamma_{j}^{2}, \frac{\gamma_{i}^{2}+\gamma_{j}^{2}}{2}\right)\right]\right\}
\end{aligned}
$$


and for $S_{y x}(k), k=0$ case, the result is

$$
\begin{aligned}
S_{y x}(0) & =\left(Z_{x}^{2}+N_{x}\right) \phi_{x}(0)-2 Z_{x} N_{x}\left[\phi_{x}(0)-\sum_{i=1}^{3} \alpha_{i} \phi_{x}\left(\gamma_{i}^{2}\right)\right] \\
+ & N_{x}\left(N_{x}-1\right)\left\{\phi_{x}(0)-\sum_{i=1}^{3} \alpha_{i}^{2}\left[\phi_{x}\left(\gamma_{i}^{2}\right)-\psi_{x}\left(\gamma_{i}^{2}, \gamma_{i}^{2}\right)\right]\right. \\
& \left.-\sum_{i \neq j}^{3} \sum_{j=1}^{3} \frac{\alpha_{i} \alpha_{j}}{\gamma_{i}^{2}-\gamma_{j}^{2}}\left[\phi_{x}\left(\gamma_{i}^{2}\right) \gamma_{j}^{2}-\phi_{x}\left(\gamma_{j}^{2}\right) \gamma_{i}^{2}\right]\right\},
\end{aligned}
$$

where the auxiliary functions $\phi(x)$ and $\psi(x)$ are defined as

$$
\begin{aligned}
& \phi_{x}\left(\gamma_{j}^{2}\right)=\frac{1}{2} \ln \left|2 \sqrt{k^{2}\left(k^{2} / 4+\omega^{2} / v^{2}+\gamma_{j}^{2}\right)-k^{2} z+z^{2}}+2 z-k^{2}\right|_{z_{2}}^{z_{1}}, \\
& \psi_{x}\left(\gamma_{i}^{2}, \gamma_{j}^{2}\right)=\left(k^{2} / 4+\gamma_{j}^{2} / 2\right) c^{-0.5} \ln \left|2 \sqrt{c\left(1+b z^{-1}+c z^{-2}\right)}+2 c z^{-1}+b\right|_{z_{2}}^{z_{1}}, \\
& z_{1}=(2 v)^{2}+k^{2} / 4+\gamma_{j}^{2}, \quad z_{2}=\omega^{2} / v^{2}+k^{2} / 4+\gamma_{j}^{2}, \\
& c=k^{2}\left(k^{2} / 4+\omega^{2} / v^{2}+\gamma_{j}^{2}\right)+\left(\gamma_{j}^{2}-\gamma_{i}^{2}\right)^{2}, \quad b=-k^{2}-2\left(\gamma_{j}^{2}-\gamma_{i}^{2}\right),
\end{aligned}
$$

and for $k=0$ the following relations are valid:

$$
\begin{aligned}
& \phi_{x}\left(\gamma_{j}^{2}\right)=\frac{1}{2} \ln \left|\frac{(2 v)^{2}+\gamma_{j}^{2}}{\omega^{2} / v^{2}+\gamma_{j}^{2}}\right|, \quad \psi_{x}\left(0, \gamma_{j}^{2}\right)=\phi_{x}(0)-\phi_{x}\left(\gamma_{j}^{2}\right), \\
& \psi_{x}\left(\gamma_{i}^{2}, 0\right)=0, \quad \psi_{x}\left(\gamma_{i}^{2}, \gamma_{i}^{2}\right)=\left[-\frac{1}{2}\left(1+z^{2} / \gamma_{i}^{2}\right)^{-1}\right]_{z=\omega / v}^{z=2 v}
\end{aligned}
$$

The Bethe high-velocity form for a random stopping power which is proportional to the ion $(x=\mathrm{i})$ or the atom $(x=\mathrm{a})$ net charge can be recognized in Eq. (B2).

\section{References}

[1] H. Bethe, Ann. Phys. 5, 325 (1930).

[2] F. Bloch, Ann. Phys. 16, 285 (1933).

[3] H. Esbensen, J.A. Golovchenko, Nucl. Phys. A 298, 382 (1978).

[4] K. Dettmann, Z. Phys. A 272, 227 (1975).

[5] J.M. Mc Guire, J.M. Stolterfoht, P.R. Simony, Phys. Rev. A 24, 97 (1981).

[6] J.H. Gillespie, Phys. Rev. A 18, 1967 (1978).

[7] Y.-K. Kim, K. Cheng, Phys. Rev. A 22, 61 (1980).

[8] T. Kaneko, Phys. Rev. A 43, 4780 (1991); Phys. Rev. A 34, 1779 (1986).

[9] M. Moneta, Nucl. Instrum. Methods Phys. Res. B 73, 474 (1993).

[10] J.A. Golovchenko, A.N. Goland, J.S. Rozner, C.E. Thorn, H.E. Wegner, H. Knudsen, C.D. Moak, Phys. Rev. B 23, 957 (1981).

[11] J.A. Golovchenko, D.E. Cox, N.A. Goland, Phys. Rev. B 26, 2355 (1982). 
[12] H.H. Mikkelsen, P. Sigmund, Phys. Rev. A 40, 101 (1989).

[13] P. Jackson, R.L. McCarty, Phys. Rev. B 6, 4143 (1972).

[14] H. Morgan, Jr., C.C. Sung, Phys. Rev. A 20, 818 (1979).

[15] D. Eisenberger, P.M. Platzman, Phys. Rev. A 2, 415 (1970).

[16] H.A. Bethe, R. Jakiv, Intermediate Quantum Mechanics, Benjamin/Cummings, Menlo Park, California 1986.

[17] E.C. Montenegro, W.E. Meyerhof, Phys. Rev. A 43, 2289 (1991).

[18] M. Moneta, to be published.

[19] H. Betz, Rev. Mod. Phys. 44, 465 (1972). 